\title{
1 Comparative analysis of inducible promoters in cyanobacteria
}

2 Anna Behle, Pia Saake, Ilka M. Axmann*

3 Institute for Synthetic Microbiology, Heinrich Heine University Duesseldorf, Duesseldorf,

4 Germany

$5 \quad$ *E-Mail: Ilka.Axmann@hhu.de | Website: http://www.synmikrobio.hhu.de

\section{Abstract}

Research in the field of synthetic biology highly depends on efficient, well-characterized

10 promoters. While great progress has been made with other model organisms such as

11 Escherichia coli, photosynthetic cyanobacteria still lag behind. Commonly used promoters

12 that have been tested in cyanobacteria show weaker dynamic range or no regulation at

13 all. Alternatives such as native metal-inducible promoters pose the problem of inducer 14 toxicity.

15 Here, we evaluate four different inducible promoters, both previously published and new, 16 using the modular plasmid pSHDY, in the model cyanobacterium Synechocystis sp. PCC 176803 - namely the vanillate-inducible promoter $P_{\text {vancc }}$, the rhamnose-inducible $P_{\text {rha, }}$ and 18 the aTc-inducible $\mathrm{P}_{\mathrm{L03}}$, and the $\mathrm{Co}^{2+-i n d u c i b l e ~} \mathrm{P}_{\text {соaT }}$. We estimate individual advantages 19 and disadvantages, as well as dynamic range and strength of each promoter in 20 comparison with well-established constitutive systems. We observed a delicate balance 21 between transcription factor toxicity and sufficient expression to obtain a dose-dependent 22 response to the inducer. In summary, we expand the current understanding and 23 employability of inducible promoters in order to facilitate the construction of more complex 24 regulatory synthetic networks, as well as more complicated biotechnological pathways for 25 cyanobacteria.

27 Keywords: Synechocystis, Inducible promoter, synthetic biology, cyanobacteria, pSHDY 
Abbreviations

32 aTc

anhydrotetracycline

33 Escherichia coli

E. coli

34 LAHG light-activated heterotrophic growth

35 RBS ribosome binding site

36 Synechocystis Synechocystis sp. PCC 6803

37 WT wild type

38 OD $_{750}$ Optical density at $750 \mathrm{~nm}$

39 TSS Transcription start site

\section{Introduction}

43 Cyanobacteria are versatile photoautotrophic organisms that are becoming more and 44 more interesting for various research applications. Due to their ability to fix carbon 45 photosynthetically, they are emerging as promising candidates for the biotechnological 46 production of different compounds, including biofuels ${ }^{1}$ and complex secondary 47 metabolites ${ }^{2}$.

48 Their ancestral relation to today's plant chloroplasts makes them important model 49 organisms for foundational research in the field of photosynthesis ${ }^{3}$. In addition to this, 50 many cyanobacteria are naturally competent and possess the ability to incorporate free

51 DNA into their genomes as well as receive conjugative plasmids, making them attractive 52 from a genetic engineering perspective ${ }^{4}$.

53 In recent years, more and more tools have been developed and characterized for diverse

54 cyanobacterial species ${ }^{5}$. This includes well-studied model organisms such as 55 Synechocystis sp. PCC 68036 ${ }^{6}$ Synechococcus elongatus PCC $7942^{7,8}$ and Anabaena 56 sp. PCC $7120^{9}$, but also some more recently discovered, fast-growing strains, such as 57 Synechococcus elongatus UTEX $2973^{10}$ or Synechococcus sp. PCC $7002^{11}$. One 58 challenging aspect when applying promoters previously established in Escherichia coli is 59 the difference in RNA polymerase architecture, which results in different binding affinities 60 and overall responses to promoter and operator regions ${ }^{12}$. 
61 Nevertheless, a range of promoters, both constitutive ${ }^{6,10}$ and inducible ${ }^{13}$, has been

62 engineered and successfully implemented in Synechocystis sp. PCC 6803 (referred to as

63 Synechocystis hereafter). These publications tend to either focus on a single promoter

64 construct with detailed work on sequence variations, or have a different core angle such

65 as metabolic engineering. For the purpose of engineering more extensive synthetic

66 regulatory networks, the availability of multiple differentially regulated promoters or

67 regulatory building blocks is essential; for comparability, they should be characterized in

68 a way that one can easily choose from depending on the application. In order to efficiently

69 fine-tune and optimize more complex systems which combine transcriptional and

70 translational output, fundamental evaluation of precise expression dynamics and strength

71 in the context of a range of constitutive promoters is highly desirable.

72 One of the most standard inducible promoter systems, which is based on the lac-operon

73 from E. coliand is inducible by the lactose analog IPTG, has been tested and implemented

74 in some cyanobacterial species. The $P_{\text {trc }}$ promoter, for example, performs well in $S$.

75 elongatus and is commonly used in many applications ${ }^{14}$. However, efforts to implement

76 similar constructs in Synechocystis have mostly failed, resulting in either extremely leaky

77 expression under non-induced conditions or little to no regulation at all ${ }^{15}$. For example,

78 Camsund et al. 2014 investigated sequence-specific repression patterns in

79 Synechocystis ${ }^{16}$. They reported a 2.3-fold induction ratio for the original $\mathrm{P}_{\text {trc }}$ promoter,

80 stating that this was likely due to insufficient levels of the repressor lacl in the cell, and

81 furthermore that previous success in $S$. elongatus was likely a result of higher expression

82 of lacl. Albers et al. 2015 investigated different IPTG-inducible constructs by modification

83 of the gap between the sigma factor binding sites ${ }^{17}$. They placed lacl under the control of

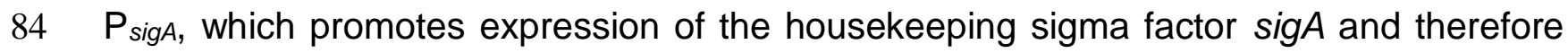

85 assures stable, strong expression of the repressor. For their promoter construct $\mathrm{P}_{\text {sca6-2, }}$

86 they were able to show approximately 10-fold induction ratios.

87 Another well-characterized promoter in Synechocystis is the aTc (anhydrotetracycline)-

88 inducible, tetR-regulated system. Huang et al. 2013 constructed a library by altering the

89 region downstream of the -10 promoter region ${ }^{18}$. They reported induction ratios of up to

90239 for their best performing promoter, PL03, under LAHG (light-activated heterotrophic

91 growth) conditions. This promoter suite was also successfully implemented by Yao et al.

922016 for dCas9-mediated gene repression, although they reported better success with the 
93 weaker, more tightly repressed $\mathrm{P}_{\mathrm{L} 22}$ due to leaky expression of dCas9 from PL03 ${ }^{19}$.

94 Unfortunately, an issue with aTc in general is the fact that it is light-degradable, making

95 its performance difficult to predict and also preventing it from stable expression under

96 photoautotrophic growth conditions, as are common for most studies focusing on

97 cyanobacteria.

98 A third system which was reported for Synechocystis by Kelly et al. in 2018 is the L-

99 rhamnose-inducible promoter $P_{r h a}$, which is regulated by the transcriptional activator

100 rhaS ${ }^{20}$. They thoroughly investigated this promoter under different light and nutrient

101 conditions and found it to be tightly repressed under non-induced conditions, with a linear

102 response upon induction and a good dynamic range, in addition to the inducer, L-

103 rhamnose, being non-toxic to and non-metabolizable by the cells. To date, this is the best

104 working promoter system in Synechocystis in terms of both performance and inducer

105 characteristics.

106 A general issue when selecting promoters for different applications is the data 107 reproducibility. Depending on factors like the choice of measurement methods, (reporter) 108 genes, RBS / 5'UTR or growth conditions, effects on mRNA stability, fold activation or 109 promoter strength may strongly differ between publications ${ }^{21-23}$. While it remains true that 110 each lab should reproduce measurements under their own conditions to ensure 111 reproducibility, an evaluation of constructs in a side-by-side manner using comparable 112 genetic elements and culturing conditions can be helpful in choosing a suitable promoter 113 to begin with.

114 In contrast to cyanobacteria, there has been ongoing, successful work published for more 115 accessible model organisms such as E. coli. For example, Ruegg et al. reported the 116 optimization of a promoter system in E. coli, previously identified in Enterobacter 117 lignolyticus ${ }^{24}$, which responds to a variety of cationic dyes at very low, non-toxic 118 concentrations, including the cheap inducer compound crystal violet, for which they report 119 a dynamic range of four orders of magnitude.

120 Another recent publication focused on optimization of parameters such as binding of the 121 transcription factor to the operator, full repression under non-induced conditions, and 122 elimination of cross-talk using a two-phase directed evolution approach ${ }^{25}$. Here, a positive 123 selection process involving expression of DNA polymerase was combined with a negative 124 selection involving the toxic expression of a mutant aminoacyl tRNA-synthetase. This 
125 yielded 12 highly optimized promoter/sensor pairs, including a vanillate-inducible system

126 originating from Caulobacter crescentus.

127 In this work, we constructed and investigated a comprehensive, comparative library of

128 available different inducible promoters by evaluating them in the same genetic

129 architecture, using the modular plasmid pSHDY. Alongside established aTc-, L-

130 rhamnose-, and $\mathrm{Co}^{2+}$-inducible systems, we also present the newly tested vanillate-

131 inducible promoter system.

132 Finally, we estimated individual promoter performance in a controlled setting for various

133 downstream applications.

\section{Results and Discussion}

\section{Design framework of all promoter constructs tested in Synechocystis.}

139 In order to assay each promoter while ensuring comparability/reproducibility, a suitable

140 reporter system was required. We considered a vector with two spatially separated cloning 141 sites, in which the reporter construct comprising promoter, RBS and reporter gene could

142 be located distantly from the repressor/activator and could easily be switched out. For this 143 reason, we constructed pSHDY, a conjugative shuttle vector based on pVZ32126, but 144 much more suitable for cloning due to multiple restriction sites flanking the antibiotic 145 resistance cassettes. In addition, pSHDY also contains the mobAY25F point mutation 146 investigated by Taton et al., which leads to an increase in supercoiled plasmid and 147 therefore more efficient downstream cloning applications such as restriction digest ${ }^{27}$.

148 The basic pSHDY cloning vector contains a total of three antibiotic resistance cassettes, 149 chloramphenicol and kanamycin, which are flanked by two independent cloning sites 150 termed the BioBrick and the NeoBrick site, respectively, and a spectinomycin resistance 151 separating the two (Fig. 1A). For the purpose of comparability, we cloned each 152 promoter/reporter construct into the BioBrick site, while keeping the corresponding 153 repressor constructs in the NeoBrick site (Fig. 1B). 


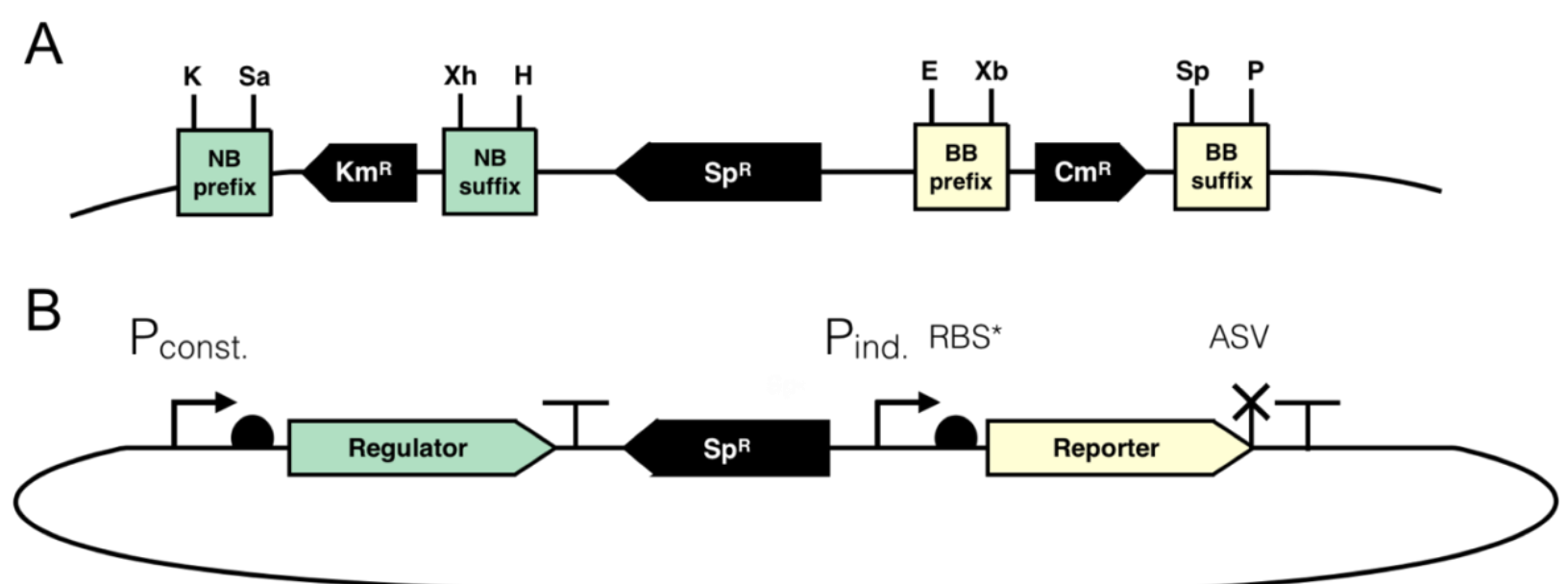

\section{pSHDY backbone}

Fig. 1: Genetic composition of the different promoter and sensor constructs measured in this work.

A: Detailed overview of the two modular cloning sites, the NeoBrick (NB) shown in green and the BioBrick (BB) sites shown in yellow. Restriction site abbreviations: K: Kpnl; Sa: Sall; Xh: Xhol; H: Hindlll; E: EcoRl; Xb: Xbal; Sp: Spel; P: Pstl B: Overview of the plasmid composition of reporter constructs used in this work. $\mathrm{P}_{\text {const: }}$ : Constitutive promoter. $\mathrm{Sp} \mathrm{R}^{\mathrm{R}}$ : Spectinomycin resistance. $\mathrm{P}_{\text {ind }}$ : inducible promoter. ASV: ssrAbased ASV-degradation tag.

164 The promoter/reporter devices were constructed in a comparable manner. For the reporter, we chose mVenus, an eYFP variant with enhanced brightness ${ }^{28}$. The gene was further codon-optimized for Synechocystis and a C-terminal ssrA-based ASV degradation tag for moderate protein turnover ${ }^{29}$ was added to prevent measuring stable protein instead of inducer-dependent expression. For the RBS, we used the established synthetic RBS ${ }^{* 30}$, which was shown to perform well in Synechocystis on multiple occasions, except in the native promoter constructs, $\mathrm{P}_{\text {coaT }}$, as well as $\mathrm{P}_{\mathrm{cpc560}}$, which has been reported to require its native $R B S$ for maximum strength ${ }^{10}$. In the case of $P_{\text {vancc, }}$, the riboJ insulator was kept

172 in the 5'UTR as was constructed in the original publication ${ }^{25}$.

174 Table 1 contains a basic description of all promoters tested in this work, while detailed 175 descriptions and sequences of each promoter construct can be found in the 176 Supplementary information (Table S1).

179 Inducible promoters are shown above, constitutive promoters below the double line. 


\begin{tabular}{|c|c|c|c|c|c|}
\hline Name & Full genotype of construct & inducer & regulator & 5'UTR & Source \\
\hline$P_{\text {vancc }}$ & $\begin{array}{l}\text { NB_PJ23100::vanR_NB } \\
\text { BB_P vancc::riboJ- } \\
\text { RBS }^{*}: \text { mVenus_BB }\end{array}$ & vanillic acid & $\operatorname{vanR}$ & riboJ + RBS* & 25 \\
\hline $\begin{array}{l}P_{\text {rha }}(111) \\
P_{r h a}(119)\end{array}$ & $\begin{array}{l}\text { NB_PJ23111::rhaS_NB } \\
\text { NB_PJ23119::rhaS_NB } \\
\text { BB_Prha::RBS }:: m \text { Venus_BB }\end{array}$ & L-rhamnose & rhas & RBS $^{*}$ & 20 \\
\hline $\begin{array}{l}\mathrm{P}_{\mathrm{L} 03}(\text { tetR-mut5) } \\
\mathrm{P}_{\mathrm{L} 03}(\text { tetR-mut8) }\end{array}$ & $\begin{array}{l}\text { NB_PJ23119-mut5::rhaS_NB } \\
\text { NB_PJ23119-mut8::rhaS_NB } \\
\text { BB_PL03::RBS*::mVenus_BB }\end{array}$ & aTc & tetR & $\mathrm{RBS}^{*}$ & 18 \\
\hline $\mathrm{P}_{\text {coat }}$ & BB_coaR_Pcoat::mVenus_BB & $\mathrm{CoCl}_{2}$ & coaR & native & Synechocystis \\
\hline $\mathrm{P}_{\text {rnpB }}$ & BB_P rnpB:::RBS*::mVenus_BB & - & 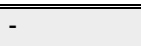 & RBS $^{*}$ & Synechocystis \\
\hline$P_{\text {cpc560 }}$ & BB_Pcpc560::mVenus_BB & - & - & native & Synechocystis ${ }^{31}$ \\
\hline PJ23119 & BB_PJ23119::RBS*::mVenus_BB & - & - & RBS $^{*}$ & BioBrick \\
\hline$P_{J 23100}$ & BB_PJ23100::RBS*::mVenus_BB & - & - & RBS $^{*}$ & BioBrick \\
\hline $\mathrm{P}_{r b c L}$ & BB_Prbcl::RBS*::mVenus_BB & - & - & $\begin{array}{l}131 \text { bp native } \\
+ \text { RBS }^{*}\end{array}$ & Synechocystis \\
\hline
\end{tabular}

181 Another consideration was encoding the repressor constructs on the genome and the 182 promoter constructs on a plasmid, but we decided against it for two reasons.

183 Firstly, the copy number of the Synechocystis genome can fluctuate depending on 184 different conditions such as growth phase, light intensity or nutrient availability, potentially 185 resulting in different repressor copy numbers and subsequent strength of gene repression. 186 In contrast, plasmid copy number is more stringently regulated within the cell, leading to 187 more consistent results ${ }^{32}$. This also relates to the fact that expression may vary depending 188 on the genomic context. Since different working groups have been using different genomic 189 integration sites, data may not be directly reproducible.

190 Secondly, it takes longer to generate fully segregated genomic mutants, extending the 191 amount of time between conceiving a project and measuring the data, further complicating 192 rapid genetic screens.

193 Therefore, we determined a plasmid-encoded reporter system to be the best option.

198 While there have been publications on vanillate inducible systems, mainly in $\alpha$ 199 proteobacteria ${ }^{33}$, but also in E. coll ${ }^{25}$, to the best of our knowledge, this is the first detailed, 200 dose-dependent promoter study in Synechocystis. One publication focusing on NOT- 
201 gates in S. elongatus PCC 7942 used the promoter/repressor pair vanR/PvanA from 202 Corynebacter glutamicum ${ }^{34}$. There, the regulation of the promoter-sensor pair was 203 successfully implemented independently of the inducer vanillate. However, vanillate204 dependent induction was not further investigated.

205 We ultimately chose to evaluate vanR/Pvancc from the recent publication by Meyer et al., $2062018^{25}$, in Synechocystis. Here, the promoter/repressor pair vanR/Pvancc from 207 Caulobacter crescentus was rationally designed and then further optimized via directed 208 evolution for E. coli, yielding a vanillate sensor with both improved dynamic range, as well 209 as lower cross-reactivity.

210 We chose the weak constitutive promoter PJ23100 from the Anderson library and the 211 published van3 RBS to control vanR, shown schematically in Fig. 2A. The van3-vanR 212 fusion was amplified from sAJM.1504, the Marionette-Clo strain (addgene ID 108251). 213 For $\mathrm{P}_{\text {vancc, }}$, we amplified the original promoter construct, including the riboJ insulator, from 214 pAJM.714 (addgene ID 108515), but replaced the RBS with the synthetic RBS*. Detailed 215 descriptions and sequences are provided in the supplementary information (Table S1).

216 The conjugative plasmid containing $P_{J 23100:: v a n R}$ and $P_{\text {vancc::mVenus were transferred }}$ 217 into Synechocystis via conjugation. Transconjugants were validated, cultured, induced 218 and mVenus fluorescence, as well as the optical density at $750 \mathrm{~nm}$, was measured in a 219 microplate reader. An empty vector control (EVC) was included for each concentration.

$22024 \mathrm{~h}$ post-induction, we observed a linear dose-response to vanillate, which saturated at 1 $221 \mathrm{mM}$ (Fig. 2B). Furthermore, under non-induced conditions, the promoter remained tightly 222 repressed, reaching the same autofluorescence levels observed in the empty vector 223 control. 

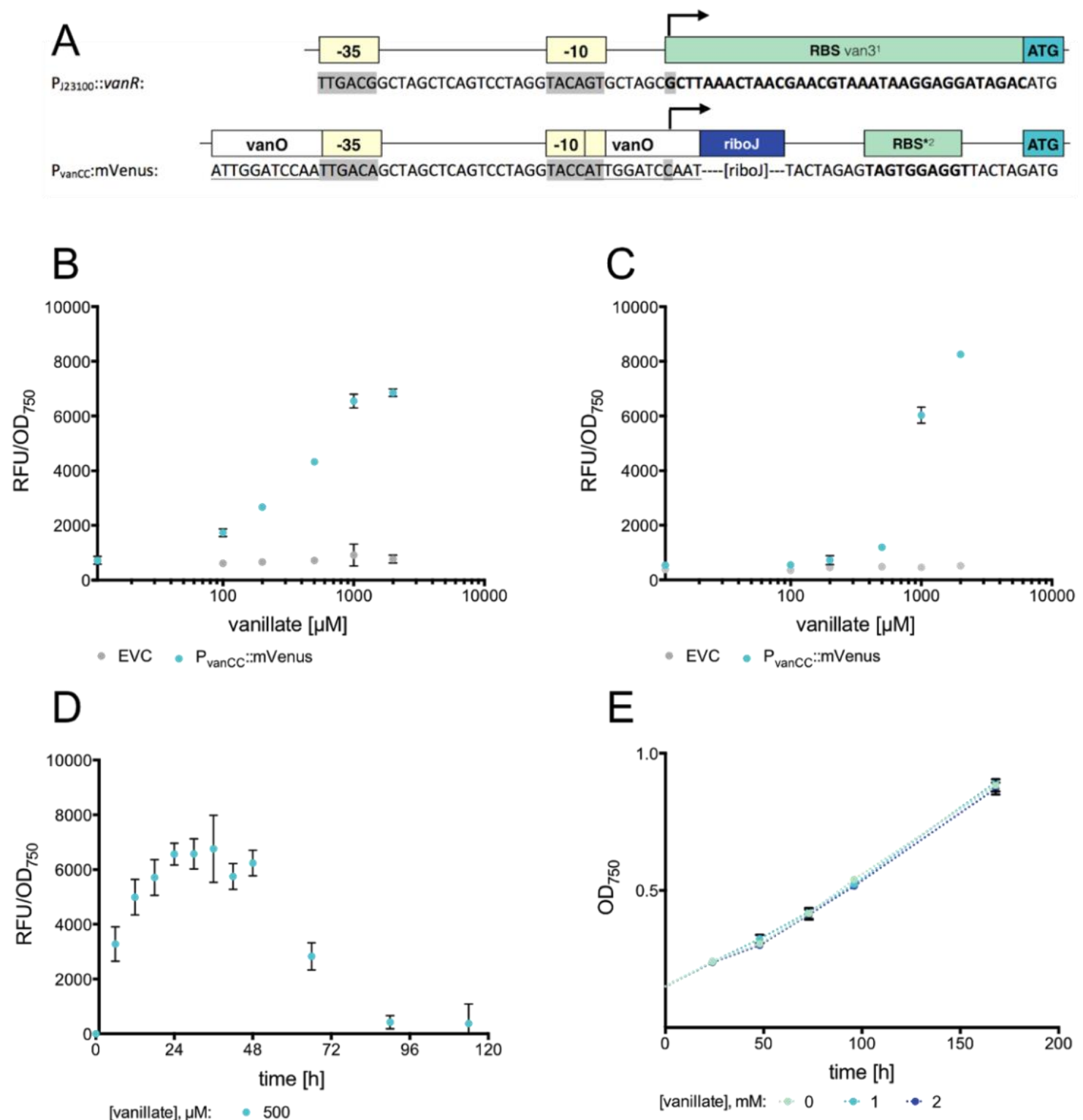

Fig. 2 Dose-dependent response of the vanillate-inducible promoter in Synechocystis.

A: Schematic overview of genetic construct used. Top: Genetic composition of regulator. Bottom: Genetic composition of regulated promoter. $-10,-35$ and +1 are highlighted in grey; RBS is shown in bold. Operator regions are underlined.

B: Dose-response of the vanillate-inducible promoter $P_{\text {vancc }}$ to different concentrations of vanillate after $24 \mathrm{~h}$

C: Dose-response of the vanillate-inducible promoter $P_{\text {vancc }}$ to different concentrations of vanillate after 48h

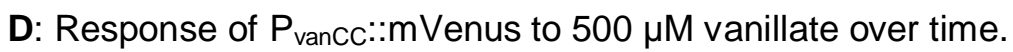
measured in a microplate reader. ${ }^{1}$ van3 RBS ${ }^{25}$; ${ }^{2}$ RBS $^{*}$ from ${ }^{30}$ 
239 After 48h, a decrease in fluorescence to a fraction of that after $24 \mathrm{~h}$ could be observed at 240 lower concentrations (100-500 $\mu \mathrm{M})$, while fluorescence increased or remained at a similar

241 level at saturating concentrations of 1-2 mM (Fig. 2C). While there is no evidence of light-

242 mediated degradation of vanillate, it is an intermediate in the biochemical degradation of

$243 \operatorname{lignin}^{35}$, so we hypothesized that vanillate might be degraded by Synechocystis after

244 longer periods of time. We therefore chose to investigate mVenus fluorescence over time.

245 To account for possible inducer degradation, we chose a vanillate concentration of 500

$246 \mu \mathrm{M}$, which was below saturation of expression and at which concentration a decrease in

247 fluorescence was observable.

248 After induction, cultures were measured every 6h. To account for effects caused by cell

249 density, an aliquot of each culture was sampled and cell density was adjusted to the start

250 OD750 of 0,25 prior to each measurement.

$251 \mathrm{P}_{\text {vancc }}$ rapidly responded to vanillate induction, reaching a fluorescence maximum 252 approximately $24 \mathrm{~h}$ post-induction. This level was maintained until $48 \mathrm{~h}$ post-induction, after 253 which fluorescence gradually decreased in a linear fashion, reaching autofluorescence 254 levels 90h post-induction (Fig. 2D).

255 Since vanillate appears to be completely degraded by Synechocystis after 90h, we chose 256 to investigate whether it had any effect on its growth. WT Synechocystis cells were treated 257 with different concentrations of vanillate, and OD750 was monitored over 7 days (Fig. 2E). 258 Interestingly, vanillate had no positive or negative influence on growth of Synechocystis.

259 We therefore hypothesize that degradation of vanillate occurs in an unspecific manner, 260 not significantly contributing as a nutrient.

261 Overall, $P_{\text {vancc }}$ performs well in Synechocystis in a dose-dependent manner, showing no 262 toxicity, tight repression and good dynamic range, with a maximum fold-induction of $16 x$ 263 (2 mM vanillate, 48h post-induction). 

expression

269 While the degradation of vanillate shown for $\mathrm{P}_{\text {vancc }}$ can be positive for certain applications,

270 it can also be a drawback when a more long-term expression is desired.

271 Since the Prha promoter published by Kelly et al. ${ }^{20}$ showed such promising results both in

272 dynamic range and stability over time, we aimed to reproduce the data. In accordance

273 with our design framework, which allows for modular exchange of parts, we chose to

274 investigate whether L-rhamnose response could be further tuned by fusing two different

275 minimal constitutive promoters upstream of the activator gene rhaS - PJ23119, containing

276 the $E$. coli consensus core elements and reportedly the strongest of the Anderson

277 promoter library, and $\mathrm{P}_{\mathrm{J} 23111}$, which was shown to be approximately half as strong as

$278 P_{\text {J23119 in Synechocystis }}{ }^{10}$ (Fig. 3A). Plasmids containing these fusions, as well as

279 Prha::mVenus, were transferred into Synechocystis via conjugation. Transconjugants were

280 validated, cultured, induced and mVenus fluorescence, as well as the OD750, was

281 measured in the plate reader. An empty vector control (EVC) was included for each

282 concentration. The strains were termed Pra::mVenus(119) or Prha::mVenus(111), with the

283 number in parentheses corresponding to the respective Anderson promoter number.

284 A typical dose-dependent response can be observed in both reporter constructs $24 \mathrm{~h}$ post 285 induction, saturating at approximately $10 \mathrm{mM}$ L-rhamnose (Fig. 3B). A maximum fold

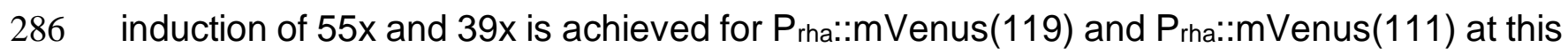

287 concentration, respectively. While Kelly et al. don't specify fold change, a 15x increase 288 under similar conditions can be roughly estimated from their data, indicating an overall 289 improvement of promoter function. We hypothesize that this improvement can be 290 attributed to both an increase of activator levels inside the cell and the use of the well291 established RBS* instead of the native E. coli RBS, which may increase the maximal 292 expression achievable with Prha. 

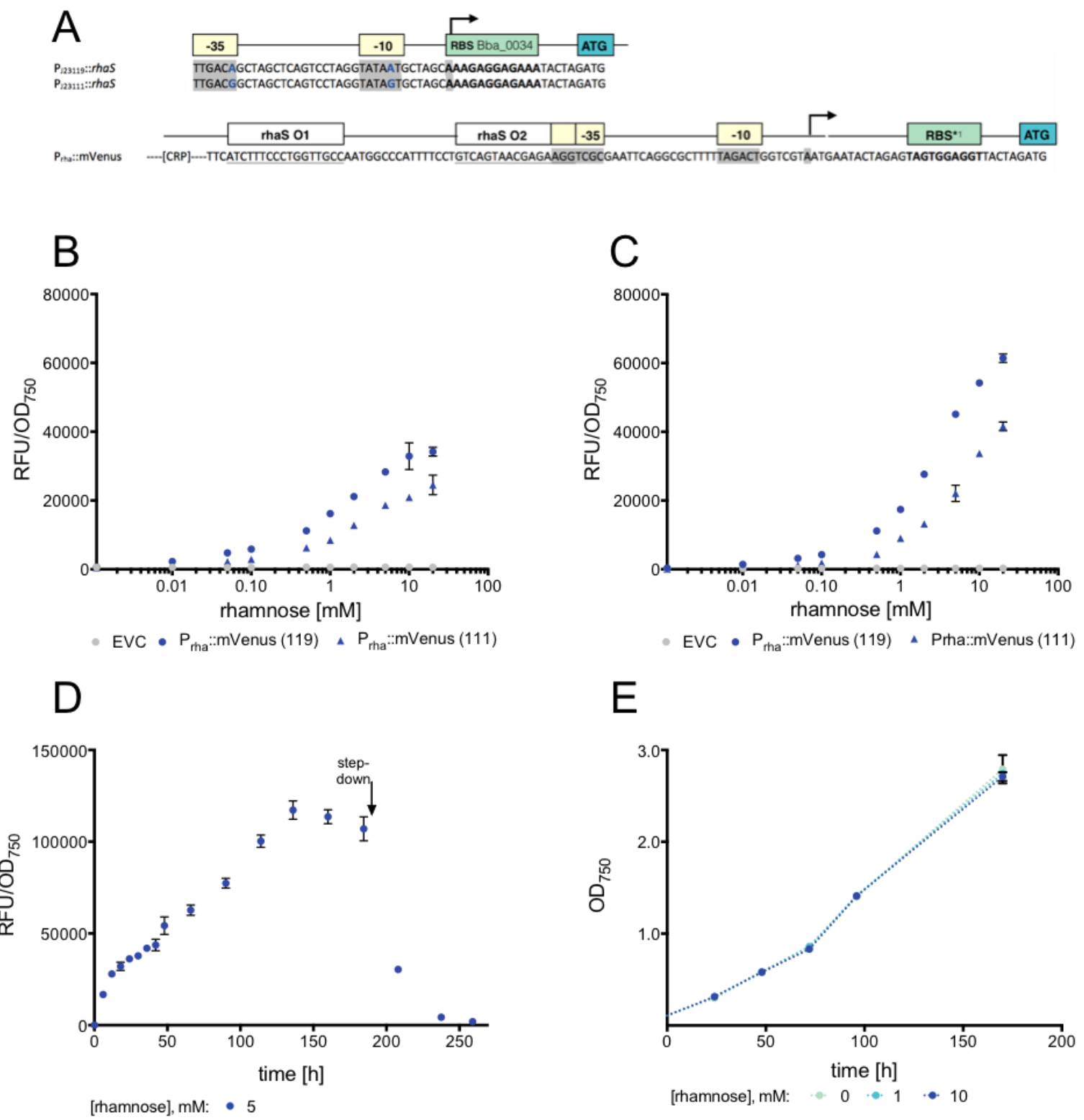

\section{Fig. 3 Dose-dependent response of the rhamnose-inducible promoter $\mathbf{P}_{\text {rha }}$ in Synechocystis.}

A: Schematic overview of genetic constructs used. Top: Genetic composition of regulator. Bottom: Genetic composition of regulated promoter $-10,-35$ and +1 are highlighted in grey; RBS is shown in bold. Operator regions are underlined.

B: Dose-response of the rhamnose-inducible promoter $P_{\text {rha }}$ to different concentrations of aTc after $24 \mathrm{~h}$

C: Dose-response of the rhamnose-inducible promoter $P_{\text {rha }}$ to different concentrations of aTc after $48 \mathrm{~h}$ to 0,25 prior to fluorescence measurement.

302 E: Growth of WT Synechocystis in different rhamnose concentrations.

303 Three biological replicates were cultured in BG11 + inducer and fluorescence and optical density was

304 measured in a microplate reader.

$305{ }^{1}$ RBS $^{*}$ from ${ }^{30}$ 
When growing induced cultures for a longer amount of time, the general dose-dependent pattern remained the same for both strains. However, both overall fluorescence intensity, as well as fold induction at $10 \mathrm{mM}$ appeared to further increase over time, up to $165 \mathrm{x}$ and 143x after $76 \mathrm{~h}$ for Prha::mVenus(119) and Prha::mVenus(111), respectively (Fig. 3C).

310 Therefore, we decided to also evaluate the short- and long-term temporal expression

311 dynamics. To account for possible inducer degradation and reliably assert expression

312 dynamics, we chose a rhamnose concentration of $5 \mathrm{mM}$, which was below saturation of

313 expression.

314 After induction, cultures were measured every 6h. To account for effects caused by cell

315 density, an aliquot of each culture was sampled and cell density was adjusted to the start

$316 \mathrm{OD}_{750}$ of 0,25 prior to each measurement.

317 Fluorescence rapidly increased directly after induction. 18h post-induction, this increase

318 became linear. Fluorescence continued to increase linearly until 136h post-induction, after

319 which fluorescence levels remained stable for three more days (Fig. 3D). To investigate

320 whether this was reversible, we performed a step-down by washing the cells twice with

321 BG11 to remove all L-rhamnose from the media. OD750 was adjusted to 1.0. Fluorescence

322 rapidly decreased after step-down, reaching pre-induction autofluorescence levels after 3 323 days.

324 Finally, we chose to investigate whether L-rhamnose had any effects on Synechocystis

325 growth, since some of the concentrations used were higher than previously tested by Kelly 326 et al. WT Synechocystis cells were treated with different concentrations of L-rhamnose, 327 and $\mathrm{OD}_{750}$ was monitored over 7 days (Fig. 3E). Consistent with previous results, L328 rhamnose had neither a positive nor a negative effect on Synechocystis growth. Moreover, 329 the fluorescence time-course results further support the hypothesis that Synechocystis is 330 unable to use L-rhamnose as a carbon source.

331 As already stated by Kelly et al., Prha performs exceptionally well as an inducible promoter, 332 with a high dynamic range, tight repression, stable expression over at least 7 days, and 333 no toxic effects or metabolization of the inducer. 
336 The aTc-inducible promoter PL03 shows improved function by increasing the protein

337 levels of the tet $R$ repressor

339 Next, we chose to evaluate the PL03 promoter published by Huang and Lindblad ${ }^{18}$. Despite

340 their promising results of $300 x$ fold induction this promoter was also reported to lead to 341 leaky expression of dCas 9 by Yao et al. ${ }^{19}$. For these reasons, we chose multiple different 342 design strategies to possibly reduce leakiness. Firstly, we removed the ssrA-based LVA 343 degradation tag to overcome rapid degradation of the repressor protein. Secondly, we 344 chose the strong promoter $\mathrm{P}_{\mathrm{J} 23119}$ in place of $\mathrm{P}_{\mathrm{J} 23101}$ to further increase intracellular TetR.

345 Finally, we applied the same plasmid-based design strategy used for the other promoters 346 to be able to compare the results later on.

347 Interestingly, we were unable to obtain clones with the expected regulatory sequences 348 upstream of tetR planned in silico. Instead, each sequenced clone showed point mutations 349 either in the promoter or RBS sequence, suggesting toxicity resulting from excessive 350 expression of tetR. Since we preselected clones that showed no fluorescence in E. coli 351 for sequencing, indicating tight repression of $\mathrm{P}_{\mathrm{L} 03}$ in E. coli, we decided to investigate two 352 of them despite the point mutations. We termed them tetR-mut5 and tetR-mut8. Fig. 4A 353 highlights the genetic composition of the two mutants compared to the desired construct.

354 Cultures containing the plasmid constructs were treated identically to the ones containing 355 the $\mathrm{P}_{\text {vancc }}$ and $\mathrm{P}_{\text {rha }}$ promoter constructs. For the purpose of employing this promoter in 356 broad, standard applications, we limited our experimental setup to photoautotrophic 357 growth conditions (see the Method section for details), despite Huang et al. reporting 358 better results for cultures grown in red light and LAHG.

359 Fig. 4B shows the dose response of the two mutant constructs $24 \mathrm{~h}$ post-induction. 360 Interestingly, the dose response assay shows the expected linear aTc-dependent 361 increase of relative fluorescence. The fold change at $1000 \mathrm{nM}$ aTc was lower than for 362 rhamnose with 16-fold and 11-fold for tetR-mut5 and tetR-mut8, respectively. While the 363 tetR-mut8 strain outperforms tetR-mut5 both in dynamic range and maximum strength of 364 the promoter in terms of relative fluorescence achieved, it also shows minimally higher 365 leaky expression under uninduced conditions (Fig. 4 B). 

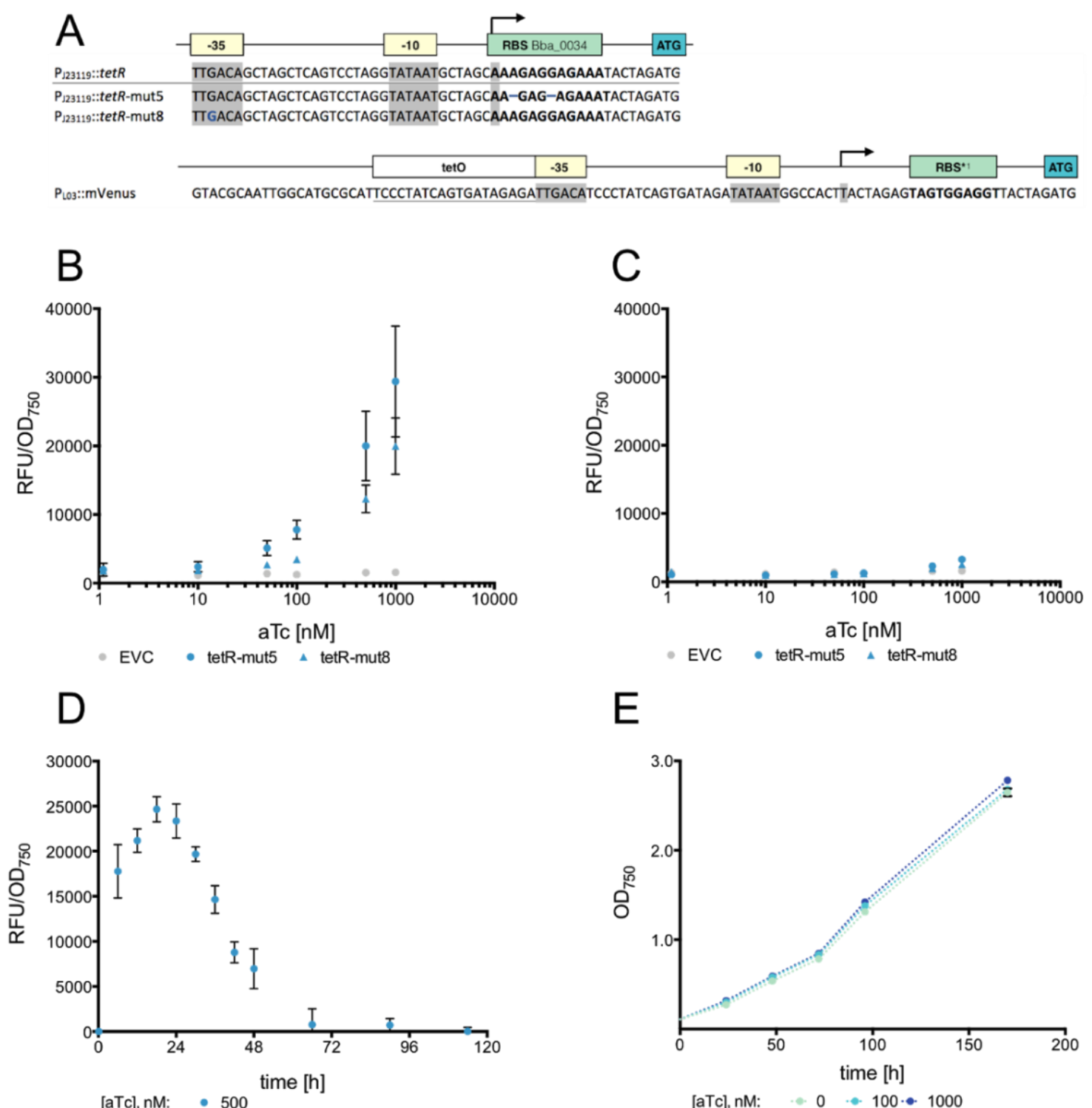

[aTc], nM: $\quad 500$
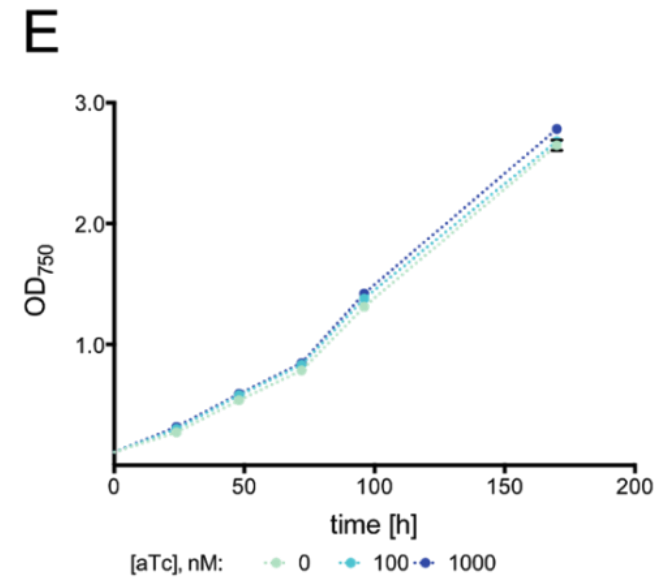

Fig. 4 Dose-dependent response of the aTc-inducible promoter $P_{\mathrm{L} 03}$ in Synechocystis.

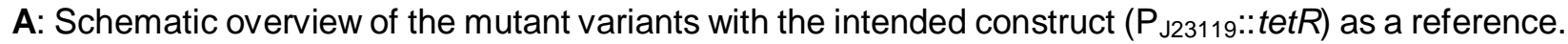
Top: Genetic composition of regulator. Bottom: Genetic composition of the regulated promoter PL03. $10,-35$ and +1 are highlighted in grey; RBS is shown in bold. Point mutations / deletions are shown in blue.

B: Dose-response of the aTc inducible promoter $\mathrm{P}_{\mathrm{L} 03}$ to different concentrations of aTc after $24 \mathrm{~h}$

D: Growth of Synechocystis WT supplemented with different concentrations of aTc. Three biological replicates each were cultured in BG11 and measured in the spectrophotometer.

376 E: Response of $\mathrm{P}_{\mathrm{L} 03}$ (tetR-mut5) to $500 \mathrm{nM}$ aTc over time. $\mathrm{OD}_{750}$ of each sample was adjusted to 0,25 377 prior to fluorescence measurement.

378 Two and three biological replicates for tetR-mut5 and tetR-mut8, respectively, were cultured in BG11

379 + inducer and fluorescence and optical density was measured in a microplate reader.

$380{ }^{1}$ RBS $^{*}$ from ${ }^{30}$ 
381 The dynamic range of mVenus expression decreased over time; by $48 \mathrm{~h}$ post-induction,

382 fluorescence had significantly decreased to a fraction of what was measured before (Fig.

383 4C). We therefore decided to also evaluate the short- and long-term temporal expression

384 dynamics.

385 To account for possible inducer degradation, we chose an aTc concentration of $500 \mathrm{nM}$, 386 which was below saturation of expression.

387 After induction, cultures were measured every 6h. To account for effects caused by cell 388 density, an aliquot of each culture was sampled and cell density was adjusted to the start

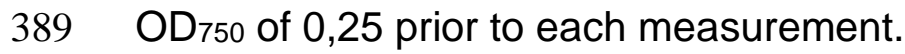

390 Consistent with the results observed for $\mathrm{P}_{\text {vancc }}$ and $\mathrm{P}_{\text {rha, }}$, fluorescence rapidly increased, 391 reaching a maximum after 18h (Fig. 4D). However, in contrast to $P_{\text {vancc, fluorescence }}$ 392 decreased again just as rapidly, reaching autofluorescence levels after 66h. In 393 accordance with published literature, the rapid decrease in fluorescence is most likely a 394 result of light-mediated degradation of aTc. As reported in Huang et al., this promoter 395 likely performs much better under LAHG in darkness or red light. However, for reasons 396 stated earlier, we chose not to further investigate PL03 under these conditions.

397 Since aTc is a derivative of the antibiotic tetracycline, there have been reports on its 398 toxicity in E. coli at high concentrations ${ }^{36}$. Thus, we were interested in its effects on the 399 growth of Synechocystis WT at the relevant concentrations used for induction of PL03.

400 Interestingly, aTc-treated cells show minimally improved growth compared to untreated 401 cells (Fig. 4E). We attribute this effect to hormesis, a positive effect on growth often 402 observed in bacteria as a result of a global stress response to sublethal concentrations of 403 antibiotics ${ }^{37}$. At concentrations relevant for the induction of $P_{L 03}$, aTc appears to have no 404 growth-inhibiting effect on Synechocystis.

405 As previously shown by Huang et al., PL03 performs well as an inducible promoter. 406 Providing a suitable intracellular amount of TetR, it shows minimal leakiness and good 407 dynamic range. Especially during the first 24h, it shows rapid, strong induction, making it 408 a suitable tool for applications within this time-frame. Due to the light-sensitive properties 409 of aTc, this promoter may be better suited under red light or darkness for longer term 410 induction experiments. It also may be beneficial for the half-life of aTc to adjust the culture 411 conditions to a higher cell density, thereby preserving the aTc due to shading. 
413 Evaluating the native $\mathbf{C o}^{2+}-$ responsive promoter, $\mathbf{P}_{c o a T}$, as an inducible promoter

415 Next, we decided to investigate a commonly used metal-inducible promoter. Since the 416 most efficient and commonly used $\mathrm{Ni}^{2+}$-responsive promoter, $\mathrm{P}_{n r s B}$, has already been 417 investigated in detail elsewhere ${ }^{13}$, we chose $\mathrm{P}_{\text {coaT. }}$. This promoter was successfully used 418 towards small-scale biotechnological production of plant terpenes ${ }^{38}$ and ethylene ${ }^{39}$ in 419 Synechocystis, as well as for mimicking a null mutant in the filamentous cyanobacterium 420 Anabaena sp. by selectively removing $\mathrm{Co}^{2+}$ and $\mathrm{Zn}^{2+}$ from the media ${ }^{40}$.

421 Since the TSS of $\mathrm{P}_{\text {coat }}$ has, to the best of our knowledge, not been mapped previously,

422 we performed 5'RACE (rapid amplification of cDNA ends) to determine the TSS of PcoaT.

423 Our results indicate at least 3 putative TSS for $\mathrm{P}_{\text {coat }}$ (Fig. 5A, Fig. S1). We therefore

424 decided to maintain the native promoter+5'UTR architecture, and fused the entire 1195

425 bp upstream of coaT, including the coaR repressor, upstream of mVenus.

426 Upon induction with different concentrations of $\mathrm{CoCl}_{2}$, a linear response could be 427 observed up to a concentration of $10 \mu \mathrm{M}$ (Fig 5B). For higher concentrations, the values

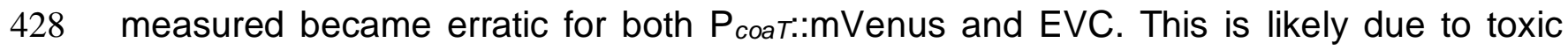
429 effects of $\mathrm{Co}^{2+}$ ions.

430 Upon investigating effects of relevant $\mathrm{CoCl}_{2}$ concentrations on growth of WT 431 Synechocystis, a slight defect in growth was observed at $10 \mu \mathrm{M}$ (Fig. 5C). This effect was

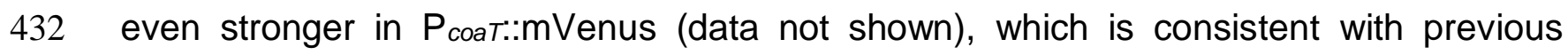
433 observations, where it was reported that deletion of coaT led to higher cobalt sensitivity ${ }^{41}$. 434 Increasing the amount of CoaR repressor in the cell, as done in this work by expression 435 of an additional copy from a plasmid, likely has the same effect. Moreover, the maximum 436 working concentration of $\mathrm{Co}^{2+}$ reported throughout the literature for the $\mathrm{P}_{\text {coat }}$ promoter is $4376 \mu \mathrm{M}^{42}$ indicating toxic effects at higher concentrations. 

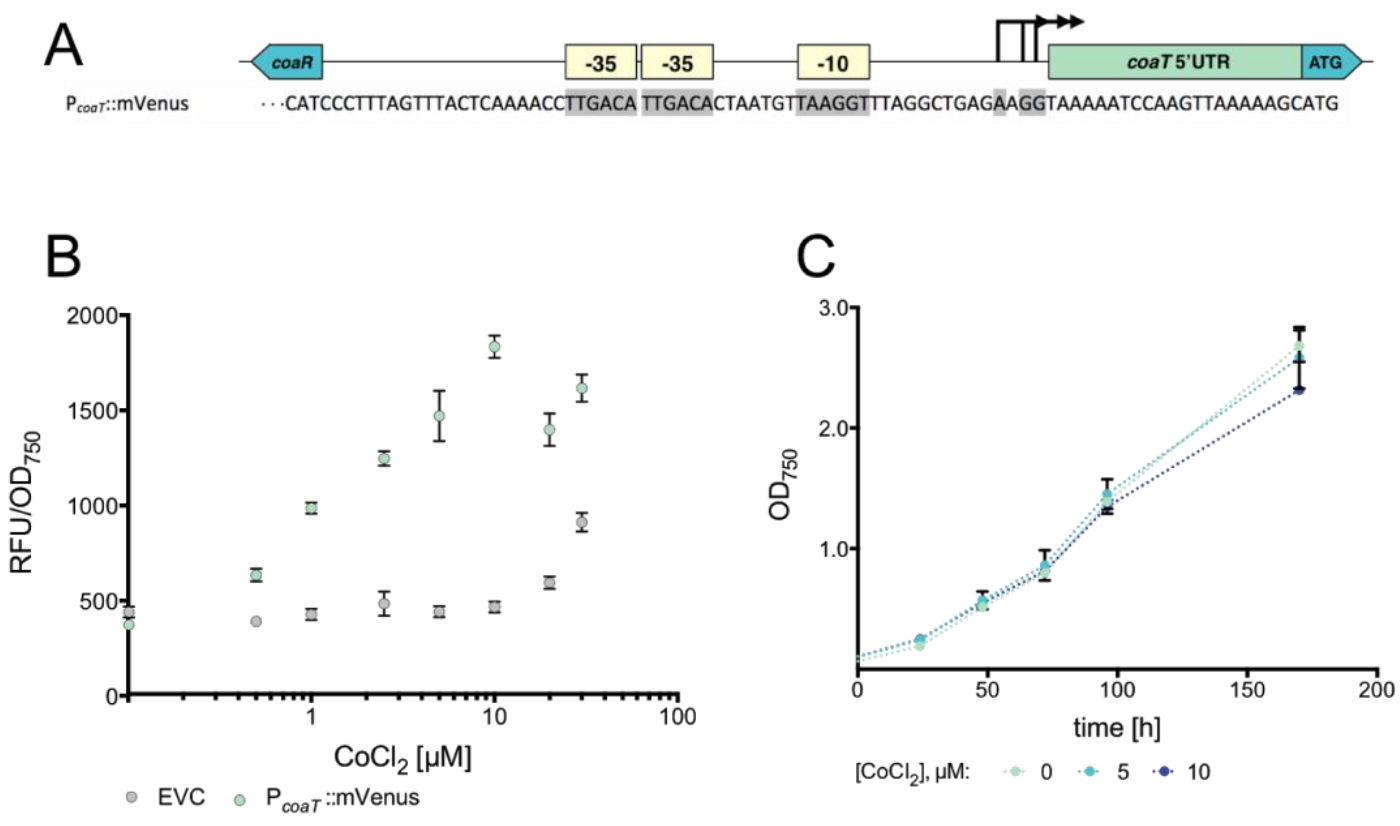

439 Fig. 5 Dose-dependent response of Synechocystis to Cobalt.

440 A: Schematic overview of genetic construct used. $-10,-35$ and putative +1 are highlighted in grey.

B: Dose-response of $\mathrm{P}_{\text {coa }}:: m$ Venus to different concentrations of $\mathrm{CoCl}_{2}$ after $48 \mathrm{~h}$

444 measured in a microplate reader.

445 More importantly, for complete repression of $\mathrm{P}_{\text {coaT }}$, it is necessary to culture strains in

$446 \mathrm{Co}^{2+}$-depleted BG11. Since $\mathrm{Co}^{2+}$ ions are required for the synthesis of coenzyme $\mathrm{B}_{12}$ in

447 diverse cyanobacteria ${ }^{43}$, this means that complete repression of the promoter may require

448 a defect in growth as a result of nutrient limitation.

449 When looking into temporal expression dynamics, Englund et al. could show a decrease

450 of fluorescence for $\mathrm{P}_{n r s B}$, due to $\mathrm{Ni}^{2+}$ actively being pumped out of the cells ${ }^{13}$. We

451 hypothesize that this is also the case for $\mathrm{Co}^{2+}$, since coaT also encodes an efflux pump.

$452 \mathrm{P}_{\text {coat }}$ specifically, as well as metal-inducible promoters in general, are unsuitable as

453 inducible promoters in synthetic biology. They lack orthogonality, require laborious

454 alteration of standard culture media, show inducer toxicity at higher concentrations and

455 are outperformed by all three inducible systems shown in this work, both in terms of

456 dynamic range and maximum strength. 
461 Finally, we measured the performance of each promoter alongside each other, either 462 uninduced or induced. In order to categorize each promoter within a broader range, we 463 included the native promoter constructs $\mathrm{P}_{\mathrm{cpc560}}, \mathrm{P}_{r n p B}$ and $\mathrm{P}_{r b c L}$, as well as the minimal 464 constitutive promoters PJ23100 and PJ23119.

465 All strains were cultured in accordance with the dose-response assays shown previously.

466 Transconjugants were validated, cultured, and mVenus fluorescence, as well as the 467 optical density at $750 \mathrm{~nm}$, was measured in a microplate reader after $24 \mathrm{~h}$. For all four 468 inducible promoters, cultures both uninduced and induced with $10 \mathrm{mM}$ L-rhamnose, $1 \mu \mathrm{M}$ 469 aTc, $1 \mathrm{mM}$ vanillate or $10 \mu \mathrm{M} \mathrm{CoCl}_{2}$, were grown and measured.

470 Consistent with the previous results, the fluorescence of $P_{\text {rha }}$ is the strongest of the 471 inducible promoters, closely followed by $\mathrm{P}_{\mathrm{L} 03}$ and $\mathrm{P}_{\mathrm{vanCC}}$ (Fig. $6 \mathrm{~A}$ ).

472 While these three show promise both in terms of dynamic range and strength, $\mathrm{P}_{\text {coat }}$ is by 473 far the weakest of the four. The uninduced control, which was cultured in regular BG11 474 instead of BG11 lacking $\mathrm{CoCl}_{2}$, shows leaky induction, leading of a fold change of only $2 x$ 475 for $\mathrm{P}_{\text {coat }}$.

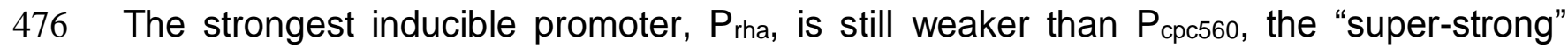
477 promoter published by Zhou et $a^{\beta 1}$. This promoter enabled expression of heterologous 478 proteins leading up to $15 \%$ of total soluble protein. However, the data shown in Fig. $6 \mathrm{~A}$ 479 was measured $24 \mathrm{~h}$ post-induction, and $P_{\text {rha }}$ shows a steady and strong increase in 480 fluorescence over 7 days (Fig. 3D). It could be assumed that $P_{\text {rha }}$ is able to reach levels 481 similar to $P_{\mathrm{cpc5} 60}$ after a sufficient induction time. 

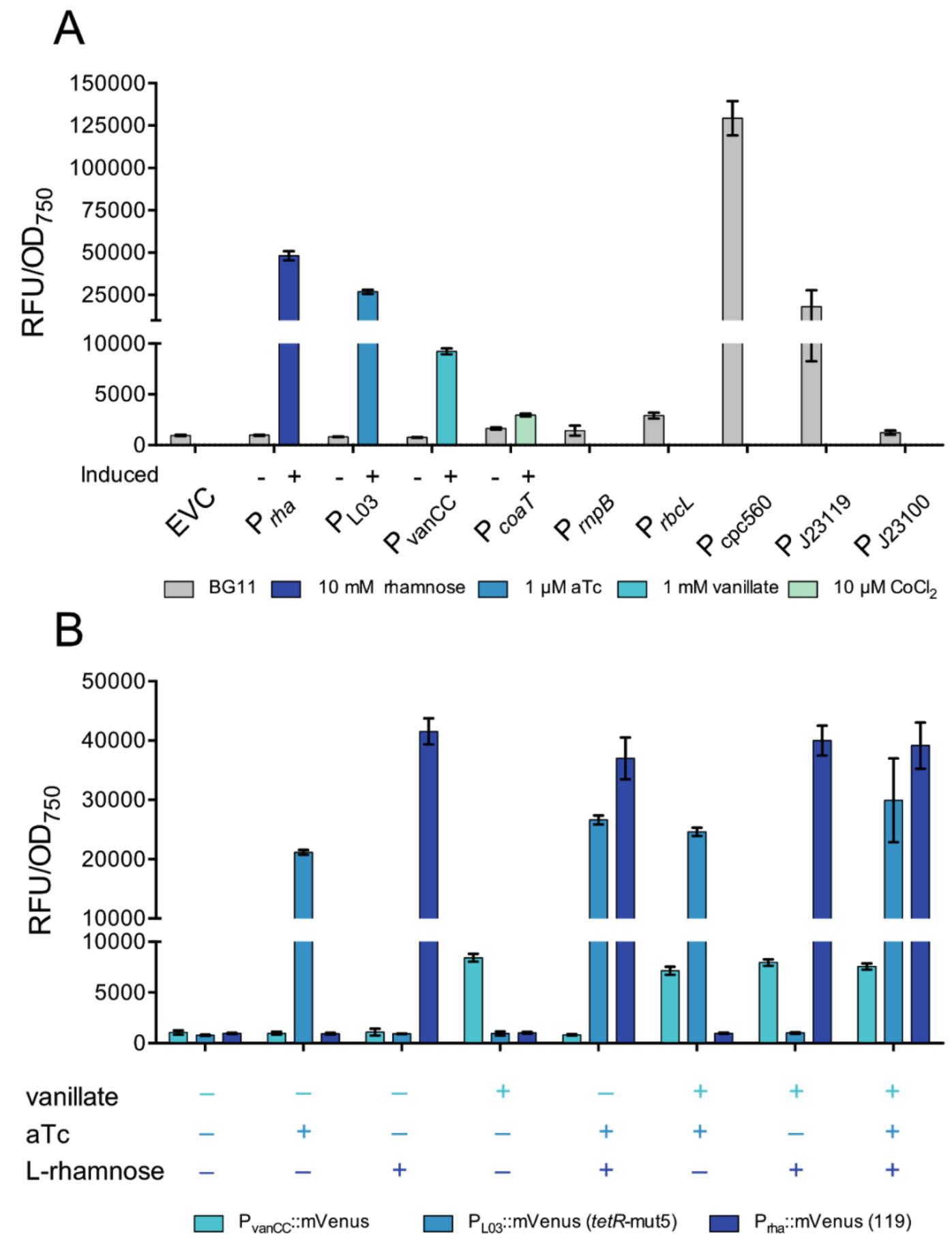

484 A: Comparison of inducible with constitutive promoters. EVC: Empty vector control. Prha: 485 Prha::mVenus(119). Pц03: PL03::mVenus (tetR-mut5). B: Evaluation of inducer specificity. or $1 \mu \mathrm{M}$ aTc or combinations thereof, marked by $\mathrm{a}+$ when present or $\mathrm{a}-$ when absent) and fluorescence and optical density was measured in a microplate reader after $24 \mathrm{~h}$. 
491 In order to investigate inducer specificity, each single promoter construct was also induced

492 with all possible combinations of inducers. Cultures were induced with $10 \mathrm{mM} \mathrm{L}$ -

493 rhamnose, $1 \mu \mathrm{M}$ aTc or $1 \mathrm{mM}$ vanillate, or a combination thereof. If left uninduced, the

494 corresponding volume of solvent $\left(\mathrm{H}_{2} \mathrm{O}\right.$ or ethanol) was added.

495 All promoters show specific induction only in the presence of the respective inducer

496 molecule (Fig. 6B). The level of fluorescence appears to be the same regardless of the

497 presence or absence of the other inducers for each promoter. In terms of inducer

498 specificity, the promoter constructs are therefore compatible with one another. It remains

499 to be investigated whether they are truly orthogonal to each other in terms of transcription

500 factor binding specificity, i.e., whether the transcriptional regulators are able to bind to

501 unspecific operator sequences and activate or repress gene expression.

503 Conclusions \& Outlook

505 In this work, we constructed and evaluated a library of different inducible promoters in a 506 way that enables a useful comparison for later selection of a suitable promoter in 507 Synechocystis sp. PCC 6803. Using the pSHDY plasmid allowed efficient exchange of 508 parts to build this library, as well as comparable conditions. We observed a delicate 509 balance between transcription factor toxicity and sufficient expression to obtain a dose510 dependent response to the inducer. This observation should be kept in mind for future 511 works, as it might significantly improve the performance of other promoters. Next to the 512 established aTc- and rhamnose-inducible promoters P 03 and Prha, we report the vanillate 513 inducible promoter $\mathrm{P}_{\text {vancc }}$ as a new tool for Synechocystis. All three promoters show a 514 linear induction over a range of inducer concentrations, as well as little to no leakiness in 515 the absence of the inducer. Interestingly, they show different strengths of expression, as 516 well as different temporal expression patterns, with the potential for a wide range of 517 biological applications. Thus, our promoter library allows moving away from metal518 inducible promoters and towards well-characterized, defined and orthogonal parts, a key 519 requirement of synthetic biology.

520 The next step in applying the three inducible promoters for future works would be 521 evaluating their performance in a strain genomically encoding the transcriptional 522 regulators. Ultimately, encoding the regulators on the genome using a markerless 
523 genomic manipulation strategy would facilitate working with cyanobacteria, since it would

524 free available space on the plasmid, as well as antibiotic resistances. This strategy has

525 proven successful in the past in E. coli, resulting in many expression strains for different

526 applications.

527 Finally, all three promoters should be combined with different reporter genes each and

528 encoded in one strain to evaluate whether they are truly orthogonal and whether they can

529 be used in combination to control multiple genes or operons, enabling the possibility for

530 larger synthetic networks or metabolic engineering by optimization of metabolic pathways.

\section{Material \& Methods}

534 Plasmid and strain construction.

535 A detailed list of all relevant genetic modules and information regarding their origin, as

536 well as plasmids constructed from them, is provided in the Supplemental Information 537 (Table S1).

538 All parts were amplified and fused using overlap extension PCR

539 (dx.doi.org/10.17504/protocols.io.psndnde) and integrated into the pSHDY backbone via 540 Gibson assembly (dx.doi.org/10.17504/protocols.io.n9xdh7n).

541 Plasmids were transferred to Synechocystis sp. PCC 6803 wild type using triparental

542 mating (dx.doi.org/10.17504/protocols.io.psndnde). Clones were verified via colony PCR

543 (dx.doi.org/10.17504/protocols.io.mk5c4y6).

545 Culture conditions.

546 All strains were maintained on BG11 plates containing $40 \mu \mathrm{g} / \mathrm{mL}$ spectinomycin.

547 Prior to each assay, BG11 $+20 \mu \mathrm{g} / \mathrm{mL}$ spectinomycin were inoculated with the strain of 548 interest, grown for 5 days, diluted to an OD750 of 0.2, grown for 3 more days, and diluted 549 again to the desired $\mathrm{OD}_{750}$ (specified in each assay) prior to starting the experiment.

550 Liquid cultures were grown in constant white light $\left(80 \mu \mathrm{mol} \cdot \mathrm{m}^{-2} \cdot \mathrm{s}^{-1}, 16 \%\right.$ intensity setting 551 in the Infors $\mathrm{HT}$ multitron) at $30{ }^{\circ} \mathrm{C}$ and $75 \%$ humidity with constant agitation at $150 \mathrm{rpm}$ 552 without added $\mathrm{CO}_{2}$.

553 Detailed protocols for each assay can be found on protocols.io:

554 Dose response assay $\rightarrow$ dx.doi.org/10.17504/protocols.io.55wg87e 
555 Toxicity assay $\rightarrow \mathrm{dx}$.doi.org/10.17504/protocols.io.6tghejw

556 Fluorescence time course assay $\rightarrow$ dx.doi.org/10.17504/protocols.io.6tkhekw

558 Measurements \& settings.

559 To determine cell density, absorbance of cells was measured in a Specord 200 Plus 560 spectrophotometer (Analytik Jena) at $750 \mathrm{~nm}$.

561 Fluorescence measurements were performed using a BMG Clariostar. Absorbance at 750 $562 \mathrm{~nm}$, as well as fluorescence at $\lambda^{\mathrm{ex}} / \lambda^{\mathrm{em}} 511 / 552$, was measured every time. Prior to each

563 measurement, the plate was shaken at $500 \mathrm{rpm}$ for 30 seconds.

564 The exact protocol for the BMG can be found in Supplementary File 1.

566 Data analysis \& -treatment.

567 For dose response assays, fluorescence values were divided by $\mathrm{OD}_{750}$.

568 For fluorescence time course assays, fluorescence values were divided by $\mathrm{OD}_{750}$. Then,

569 the mean of the values measured for the uninduced control culture was subtracted from 570 each individual value measured for the induced culture.

571 For the fluorescence time courses, all raw fluorescence values were normalized to $\mathrm{OD}_{750 \text {, }}$ 572 then, the mean fluorescence of the uninduced control was subtracted from each value of 573 the induced culture.

575 The plasmid pAJM.714, as well as the strain sAJM.1504 were a gift from Christopher Voigt 576 (Addgene plasmid \# 108515; Bacterial strain \# 108251).

Author Contributions:

$579 \quad A B$ and IMA designed and conceived the study. $A B$ and PS performed the experiments

580 and analyzed the data. AB wrote the manuscript with input from PS and IMA.

582 Conflict of Interest Disclosure:

583 The authors declare no conflict of interest. 
References

(1) Oliver, N. J., Rabinovitch-Deere, C. A., Carroll, A. L., Nozzi, N. E., Case, A. E., and Atsumi, S. (2016) Cyanobacterial metabolic engineering for biofuel and chemical production. Current Opinion in Chemical Biology 35, 43-50. (2) Ducat, D. C., Way, J. C., and Silver, P. A. (2011) Engineering cyanobacteria to generate high-value products. Trends in Biotechnology 29, 95-103. (3) Zarzycki, J., Axen, S. D., Kinney, J. N., and Kerfeld, C. A. (2013) Cyanobacterialbased approaches to improving photosynthesis in plants. J. Exp. Bot. 64, 787-798. (4) Ramey, C. J., Barón-Sola, A., Aucoin, H. R., and Boyle, N. R. (2015) Genome Engineering in Cyanobacteria: Where We Are and Where We Need To Go. ACS Synth. Biol. 4, 1186-1196.

(5) Huang, H.-H., Camsund, D., Lindblad, P., and Heidorn, T. (2010) Design and characterization of molecular tools for a Synthetic Biology approach towards developing cyanobacterial biotechnology. Nucleic Acids Research 38, 2577-2593.

(6) Wang, B., Eckert, C., Maness, P.-C., and Yu, J. (2018) A Genetic Toolbox for Modulating the Expression of Heterologous Genes in the Cyanobacterium

603

604

605 Synechocystis sp. PCC 6803. ACS Synth. Biol. 7, 276-286.

\section{(7) Kim, W. J., Lee, S.-M., Um, Y., Sim, S. J., and Woo, H. M. (2017) Development of} SyneBrick Vectors As a Synthetic Biology Platform for Gene Expression in Synechococcus elongatus PCC 7942. Front. Plant Sci 8, 1-9.

(8) Santos-Merino, M., Singh, A. K., and Ducat, D. C. (2019) New Applications of Synthetic Biology Tools for Cyanobacterial Metabolic Engineering. Front. Bioeng. Biotechnol. 7, 273.

(9) Higo, A., and Ehira, S. (2019) Spatiotemporal Gene Repression System in the Heterocyst-Forming Multicellular Cyanobacterium Anabaena sp. PCC 7120. ACS Synth. Biol. 8, 641-646.

612 (10) Vasudevan, R., Gale, G. A. R., Schiavon, A. A., Puzorjov, A., Malin, J., Gillespie, M. D., Vavitsas, K., Zulkower, V., Wang, B., Howe, C. J., Lea-Smith, D. J., and McCormick, A. J. (2019) CyanoGate: A Modular Cloning Suite for Engineering Cyanobacteria Based on the Plant MoClo Syntax. Plant Physiology 180, 39-55. (11) Markley, A. L., Begemann, M. B., Clarke, R. E., Gordon, G. C., and Pfleger, B. F. (2014) Synthetic Biology Toolbox for Controlling Gene Expression in the Cyanobacterium Synechococcus sp. strain PCC 7002. ACS Synth. Biol. 4, 595-603. (12) Imamura, S., and Asayama, M. (2009) Sigma Factors for Cyanobacterial Transcription. Gene Regulation and Systems Biology 1-23. (13) Englund, E., Liang, F., and Lindberg, P. (2016) Evaluation of promoters and ribosome binding sites for biotechnological applications in the unicellular cyanobacterium Synechocystis sp. PCC 6803. Sci. Rep. 6, 36640. (14) Geerts, D., Bovy, A., De Vrieze, G., Borrias, M., and Weisbeek, P. (1995) Inducible expression of heterologous genes targeted to a chromosomal platform in the cyanobacterium Synechococcus sp. PCC 7942. Microbiology 141, 831-841. (15) Ferreira, E. A., Pacheco, C. C., Pinto, F., Pereira, J., 2018. Expanding the toolbox for Synechocystis sp. PCC 6803: validation of replicative vectors and characterization of a novel set of promoters. Synthetic Biology. 
630 (16) Camsund, D., Heidorn, T., and Lindblad, P. (2014) Design and analysis of Laclrepressed promoters and DNA-looping in a cyanobacterium. Journal of Biological

632 Engineering 8, 1-23.

633 (17) Albers, S. C., Gallegos, V. A., and Peebles, C. A. M. (2015) Engineering of genetic control tools in Synechocystis sp. PCC 6803 using rational design techniques. Journal of Biotechnology 216, 36-46.

(18) Huang, H.-H., and Lindblad, P. (2013) Wide-dynamic-range promoters engineered for cyanobacteria. Journal of Biological Engineering 7, 1-11. (19) Yao, L., Cengic, I., Anfelt, J., and Hudson, E. P. (2016) Multiple Gene Repression in Cyanobacteria Using CRISPRi. ACS Synth. Biol. 5, 207-212.

640 (20) Kelly, C. L., Taylor, G. M., Hitchcock, A., Torres-Méndez, A., and Heap, J. T. (2018) A Rhamnose-Inducible System for Precise and Temporal Control of Gene Expression in Cyanobacteria. ACS Synth. Biol. 7, 1056-1066.

643 (21) Thiel, K., Mulaku, E., Dandapani, H., Nagy, C., Aro, E.-M., and Kallio, P. (2018) Translation efficiency of heterologous proteins is significantly affected by the genetic context of RBS sequences in engineered cyanobacterium Synechocystis sp. PCC 6803. Microbial Cell Factories 17, 1-12.

(22) Cho, S. H., Haning, K., Shen, W., Blome, C., Li, R., Yang, S., and Contreras, L. M. (2017) Identification and Characterization of 5' Untranslated Regions (5'UTRs) in

650 (23) Los, D. A., Zorina, A., Sinetova, M., Kryazhov, S., Mironov, K., and Zinchenko, V. V. (2010) Stress Sensors and Signal Transducers in Cyanobacteria. Sensors 10, 23862415.

(24) Ruegg, T. L., Pereira, J. H., Chen, J. C., Nature, A. D., 2018. Jungle Express is a versatile repressor system for tight transcriptional control. Nature Communications. (25) Meyer, A. J., Segall-Shapiro, T. H., Glassey, E., Zhang, J., and Voigt, C. A. (2019) Escherichia coli "Marionette" strains with 12 highly optimized small-molecule sensors. Nature Chemical Biology 15, 196-204. (26) Zinchenko, V. V., Piven, I. V., Melnik, V. A., and Shestakov, S. V. (1999) Vectors for the Complementation Analysis of Cyanobacterial Mutants. Russian Journal of Genetics 35, 228-232.

663 (27) Taton, A., Unglaub, F., Wright, N. E., Zeng, W. Y., Paz-Yepes, J., Brahamsha, B., Palenik, B., Peterson, T. C., Haerizadeh, F., Golden, S. S., and Golden, J. W. (2014) Broad-host-range vector system for synthetic biology and biotechnology in cyanobacteria. Nucleic Acids Research 42, e136-e136.

668 (29) Andersen, J. B., Sternberg, C., Poulsen, L. K., Bjorn, S. P., Givskov, M., and Molin, S. (1998) New unstable variants of green fluorescent protein for studies of transient gene expression in bacteria. Applied and Environmental Microbiology 64, 2240-2246. (28) Kremers, G.-J., Goedhart, J., van Munster, E. B., and Gadella, T. W. J. (2006) Cyan and yellow super fluorescent proteins with improved brightness, protein folding, and FRET Förster radius. Biochemistry 45, 6570-6580.

674 (31) Zhou, J., Zhang, H., Meng, H., Zhu, Y., Bao, G., Zhang, Y., Li, Y., and Ma, Y. 675 (2014) Discovery of a super-strong promoter enables efficient production of

676 heterologous proteins in cyanobacteria. Sci. Rep. 4, 235-6. 
(32) Rawlings, D. E., and Tietze, E. (2001) Comparative Biology of IncQ and IncQ-Like Plasmids. Microbiology and Molecular Biology Reviews 65, 481-496. (33) Kaczmarczyk, A., Vorholt, J. A., and Francez-Charlot, A. (2014) Synthetic vanillateregulated promoter for graded gene expression in <i>Sphingomonas</i>. Sci. Rep. 4 , 6453.
(34) Taton, A., Ma, A. T., Ota, M., Golden, S. S., and Golden, J. W. (2017) NOT Gate Genetic Circuits to Control Gene Expression in Cyanobacteria. ACS Synth. Biol. 6, 2175-2182. (35) Kamimura, N., Takahashi, K., Mori, K., Araki, T., Fujita, M., Higuchi, Y., and Masai, E. (2017) Bacterial catabolism of lignin-derived aromatics: New findings in a recent decade: Update on bacterial lignin catabolism. Environmental Microbiology Reports 9 , 679-705. (36) Oliva, B., Gordon, G., McNicholas, P., Ellestad, G., and Chopra, I. (1992) Evidence that tetracycline analogs whose primary target is not the bacterial ribosome cause lysis of Escherichia coli. Antimicrobial Agents and Chemotherapy 36, 913-919. (37) Mathieu, A., Fleurier, S., Frénoy, A., Dairou, J., Bredeche, M.-F., Sanchez-Vizuete, P., Song, X., and Matic, I. (2016) Discovery and Function of a General Core Hormetic Stress Response in E. coli Induced by Sublethal Concentrations of Antibiotics. CellReports 17, 46-57.

(38) Loeschcke, A., Dienst, D., Wewer, V., Hage-Hülsmann, J., Dietsch, M., KranzFinger, S., Hüren, V., Metzger, S., Urlacher, V. B., Gigolashvili, T., Kopriva, S., Axmann, I. M., Drepper, T., and Jaeger, K.-E. (2017) The photosynthetic bacteria Rhodobacter capsulatus and Synechocystis sp. PCC 6803 as new hosts for cyclic plant triterpene biosynthesis. PLOS ONE 12, e0189816. Ethylene Synthesis and Regulated Expression of Recombinant Protein in Synechocystis sp. PCC 6803. PLoS ONE (Neilan, B., Ed.) 7, e50470-11.

(40) González, A., Bes, M. T., Peleato, M. L., and Fillat, M. F. (2016) Expanding the Role of FurA as Essential Global Regulator in Cyanobacteria. PLoS ONE (Hess, W. R., Ed.) 11, e0151384.

(41) Cavet, J. S., Borrelly, G. P. M., and Robinson, N. J. (2003) Zn, Cu and Co in cyanobacteria: selective control of metal availability. FEMS Microbiol Rev 27, 165-181. (42) Berla, B. M., Saha, R., Immethun, C. M., Maranas, C. D., Moon, T. S., and Pakrasi, H. (2013) Synthetic biology of cyanobacteria: unique challenges and opportunities. Front. Microbiol. 4.

712 (43) Holm Hansen, O., Gerloff, G. C., and Skoog, F. (1954) Cobalt as an Essential 